\title{
DESIGNING OPTIMIZED TERNARY CATALYTIC ALLOY ELECTRODE FOR EFFICIENCY IMPROVEMENT OF SEMICONDUCTOR GAS SENSORS USING A MACHINE LEARNING APPROACH
}

\section{Suman Ghosal ${ }^{1 *}$, Swati Dey², Partha Pratim Chattopadhyay ${ }^{3}$, Shubhabrata Datta ${ }^{4}$ and Partha Bhattacharyya ${ }^{1}$}

\footnotetext{
${ }^{1}$ Department of Electronics and Telecommunication Engineering, Indian Institute of Engineering Science and Technology, Shibpur, West Bengal, India.

${ }^{2}$ Department of Aerospace Engineering and Applied Mechanics, Indian Institute of Engineering Science and Technology, Shibpur, West Bengal, India.

${ }^{3}$ National Institute of Foundry and Forge Technology, Hatia, Ranchi 834003, India.

${ }^{4}$ Department of Mechanical Engineering, SRM Institute of Science and Technology, Kattankulathur, India.
}

Received: 19 December 2020;

Accepted: 17 May 2021;

Available online: 13 June 2021.

Original scientific paper Abstract: Catalytic noble metal (s) or its alloy (s) has long been used as the electrode material to enhance the sensing performance of the semiconducting oxide-based gas sensors. In the present paper, optimized ternary metal alloy electrode has been designed, while the database is in pure or binary alloy compositions, using a machine learning methodology is reported for detection of $\mathrm{CH}_{4}$ gas as a test case. Pure noble metals or their binary alloys as the electrode on the semiconducting $\mathrm{ZnO}$ sensing layer were investigated by the earlier researchers to enhance the sensitivity towards $\mathrm{CH}_{4}$. Based on those research findings, an artificial neural network (ANN) model was developed considering the three main features of the gas sensor devices, viz. response magnitude, response time and recovery time as a function of $\mathrm{ZnO}$ particle size and the composition of the catalytic alloy. A novel methodology was introduced by using ANN models considered for optimized ternary alloy with enriched presentation through the multi-objective genetic algorithm (GA) wherever the generated Pareto front was used. The prescriptive data analytics methodology seems to offer more or less convinced evidence for future experimental studies.

*Corresponding author.

E-mail addresses: ghosal.suman987@gmail.com (S.Ghosal), swatidey@yahoo.com (S.Dey), ppc@metal.becs.ac.in (P.P.Chattopadhyay), shu.datt@gmail.com (S.Datta), pb_etc_besu@yahoo.com (P. Bhattacharyya) 
Designing optimized ternary catalytic alloy electrode for efficiency improvement of semiconductor gas sensors using a machine learning approach

Keywords: Oxide based gas sensor, Ternary alloy catalyst design, Sensing parameters, Artificial neural network, Genetic algorithm, Multi-objective optimization.

\section{Introduction}

Noble metals like palladium, platinum, silver, gold etc. were investigated for a long time due to their contribution towards improving the performance of semiconductor gas sensor devices (Acharyya et al., 2016). It was found that the catalytic activities of these metals can further be reinforced by judiciously alloying it with a secondary metal (Acharyya \& Bhattacharyya, 2016; Roy et al., 2012). Such binary alloys, often utilized in the form of electrode, act as the potential adsorption size for the target gas species, either through chemical sensitization or through electronic sensitization (Roy et al., 2012). They help in lowering down the activation energy requirement for gas dissociation. Due to subsequent spill-over effect, the surface activity of the main sensing layer (i.e., semiconducting oxide) is also significantly enhanced, which often leads to lower operating temperature, better sensitivity and faster response/recovery kinetics of the device (Acharyya \& Bhattacharyya, 2016; Roy et al., 2012; Quaranta et al., 1999; Bhattacharyya et al., 2007; Bhattacharyya et al., 2015). Moreover, in some cases, the alloying element often retards the degradation of the catalytic metal electrodes, thereby improving the long-term stability of the device dramatically (Roy et al., 2012; Quaranta et al., 1999; Bhattacharyya et al., 2007; Bhattacharyya et al., 2015; Wollenstein et al., 2003; Lee et al., 2003). For example, Pd electrode on $\mathrm{ZnO}$ sensing layer was found to offer improved sensitivity, but at the cost of poor stability towards $\mathrm{CH}_{4}$ (Bhattacharyya et al., 2008; Bhattacharyya et al., 2007; Basu et al., 2008). As long-term exposure to $\mathrm{CH}_{4}$ often leads to formation of Palladium hydrate which, due to lattice mismatch with $\mathrm{ZnO}$, degrades the stability of the sensor (particularly at high temperature) (Bhattacharyya et al., 2007). Further studies revealed that if $\mathrm{Pd}$ is alloyed with $25-30 \%$ of $\mathrm{Ag}$, the probability of such hydrate formation is reduced significantly, which leads to better stability of the metal semiconductor junction and the device as a whole (Maity et al., 2018). However, not a large variety of such binary alloys on different oxide surface has so far been investigated (Bhattacharyya et al., 2008; Basu et al., 2008; Mishra et al., 2010; Bhattacharyya et al., 2008; Ghosal et al., 2019). Moreover, most of the approaches are based on trial-and-error method which is time consuming, expensive, and even without any guarantee of success.

To improve the performance of such catalytic electrode material, computational design of the alloy, before experimentation, is of immense importance to avoid the above limitations. The non-availability of constitutive models for complex materials systems has prompted researchers to rely on data-driven design approaches (Datta \& Chattopadhyay, 2013). However, the artificial intelligence (AI) and machine learning (ML) have been found to be effective tools for the purpose of designing the alloys, using the experimental findings published by the earlier researchers (Datta \& Chattopadhyay, 2013). Kumar et al. (2011) used ML techniques on the raw data attained from four different odours/gases, responses of an oxygen plasma treated thick film tin oxide sensor array. Pławiak and Rzecki (2015) employed similar methods to study the effect of gas concentration on the performance of a sensor. In an earlier work, by the present authors (Ghosal et al., 2019) aimed at oxide-based gas sensor to sense methane gas competently, AI based methodology was incorporated 
magnificently to design ternary catalytic alloy systematically as per the data set of pure or binary alloys. This was the first attempt to design ternary electrode materials using AI. However, in that work, the ternary alloys were designed without any constraint in the combinations (weight percentage) of elements in the alloys or the amount of each element in it. On the contrary, in the present work, a noble approach has been employed using AI techniques to design ternary alloy catalysts with improved performance, where the experience of the earlier researchers in selection of elements and the maximum and the minimum allowable limit on the amount of a particular alloy element was incorporated in the database through restructuring the data, and thus incorporating the system knowledge in the models (The method of data restructuring is explained in database section).

The objectives of the present work are to improve the three pivotal performance parameters of the gas sensor device, viz. response magnitude, response time and recovery time, simultaneously. As the objectives (performance parameters of the sensors) are repeatedly contradictory in nature, multi-objective optimization using genetic algorithm (GA) has been employed for designing alloys with conflicting requirement (Deb, 2001; Goldberg, 2002; Dey et al., 2016; Datta, 2016). As we have already mentioned above, a model through the data made by the past researchers on binary alloys was established for three different aspects through artificial neural network (ANN) (Kumar, 2004; Anderson, 1995). ANN has proven its capability to plot the input-output relationship of compound materials systems (Longo et al., 2017; Ray et al., 2009) aimed at optimization process. The ANN models are used as the objective functions. The ANN models as objective functions while using for GA based optimizations techniques in materials structures had been fruitfully designed by the earlier researchers (Datta \& Chattopadhyay, 2013; Sinha et al., 2013). While developing the Pareto fronts (Goldberg, 2002) as a consequence in the optimization procedure comprising non-dominated solutions was established for identifying the nature of combinations and structures to design the optimized alloy with potential to improve the gas sensor performance in a predetermined and tailor-made fashion.

\section{Problem formulation}

The traditional trial and error methodology of designing new materials particularly, binary or ternary complex, with improved performance is a timewasting process, which might often lead to a whole sewerage of materials. The concept of designing computational materials using intelligent data analytics techniques can search solutions computationally, which can later be experimentally validated. In the present case, three attributes, viz. response magnitude, response time and recovery time, were used via the measure of performance of the gas sensor. These attributes were described as the compositional utilities of the catalytic metal electrode, particle size of $\mathrm{ZnO}, \mathrm{CH}_{4}$ concentrations and the optimum temperature of sensing (Quaranta et al., 1999). The designed catalyst alloy was targeted to overcome the issues related to the slow response and recovery kinetics, and not higher response magnitude. Due to this, for enhancing the device performance can be defined as dropping the response time and recovery time and increasing the response magnitude simultaneously. As revealed from the earlier reports, these objects might often be contradictory and that is why multi-objective optimization through the GA was engaged in the present approach. For describing the overhead three aspects, three distinct ANN models were developed, were recycled, and used as objective functions for studying the optimization techniques. As multi- objective complications do not lead to single optimum solution, non-dominant solutions were achieved, proposing 
Designing optimized ternary catalytic alloy electrode for efficiency improvement of semiconductor gas sensors using a machine learning approach

the best suitable cooperation between the objectives, known as Pareto front (Dey et al., 2015). These solutions can be analyzed and utilized to design optimized ternary alloy electrode, suitable for enhancing the performance of semiconducting oxidebased gas sensor devices.

\section{Construction of database}

As mentioned earlier, the database was generated from experimental results published by the earlier researchers (Bhattacharyya et al., 2008; Basu et al., 2008; Mishra et al., 2010; Bhattacharyya et al., 2008; Ghosal et al., 2019). Primary analysis of the database revealed that the noble metals used as catalytic electrode can be put into three clusters, pure metals, binary alloys with $\sim 50-50$ ratio of the elements and binary alloys with $\sim 75-25$ ratio of elements.

Table 1. The list of inputs parameters for three different output variables with their minimum, maximum, average and standard deviation (Bhattacharyya et al., 2008; Basu et al., 2008; Mishra et al., 2010; Bhattacharyya et al., 2008; Ghosal et al., 2019).

\begin{tabular}{ccccc}
\hline Input variables & Min & Max & Mean & Standard Deviation \\
\hline CH4 conc. (\%) & 0.01 & 1.5 & 0.524754 & 0.426081395 \\
\hline Temperature (oC) & 100 & 350 & 225.4098 & 64.54517205 \\
\hline ZnO Particle Size (nm) & 20 & 60 & 57.72131 & 15.47054314 \\
\hline Pt (wt\%) & 0 & 100 & 8.196721 & 27.65912729 \\
\hline Pd (wt\%) & 0 & 74 & 34.42623 & 31.57660474 \\
\hline Rh (wt\%) & 0 & 100 & 22.95082 & 42.40063924 \\
\hline Ag (wt\%) & 0 & 70 & 27.86885 & 27.30779828 \\
\hline Au (wt\%) & 0 & 100 & 6.557377 & 24.95898275 \\
\hline Output variable & & & & \\
\hline Response Magnitude (\%) & 20 & 83.6 & 45.93888 & 18.74628351 \\
\hline Response Time (S) & 2.69 & 86 & 36.11855 & 18.91098114 \\
\hline Recovery Time (S) & 16 & 102 & 55.48555 & 22.39324979 \\
\hline
\end{tabular}

Aimed at designing ternary alloy, the database was derived by dividing the compositional parameters (or components) into three different components, based on the weight percent (\%) of $\mathrm{Pt}, \mathrm{Pd}, \mathrm{Rh}, \mathrm{Au}$ and $\mathrm{Ag}$ while they are used as pure or binary form as an electrode in the device via taking the clue from the experience of alloy development by the earlier researchers (Bhattacharyya et al., 2008; Basu et al., 2008; Mishra et al., 2010; Bhattacharyya et al., 2008). Among the three components of the elements, component 1 consists of the elements having more than 50 weight $\%$ in the alloy including the pure metals, component 2 consists the presence of metals within the range of 30 to 50 weight\%, and component 3 has the elements with less than 30 weight $\%$ in the alloy. This restructuring of the database was carried out to divide the elements into three groups, as per the stoichiometry maintained by earlier researchers. Such division of the alloying elements into three components, made the 
database suitable for designing ternary alloy(s). After division of the elements, it was observed that for designing ternary alloy (as per their atomic number) three probable arrangements may be made, i.e. Pt-Pd-Ag, $\mathrm{Au}-\mathrm{Pd}-\mathrm{Ag}$ and $\mathrm{Rh}-\mathrm{Pd}-\mathrm{Ag}$. In this way, the basic components of the three alloy systems could be finalized using the prior understanding of the alloys used for the purpose. In addition to above, three components, ZnO particles size, optimum temperature for sensing and the concentration of methane $\left(\mathrm{CH}_{4}\right)$ were also included as input parameters. Basically, the three output parameters are response magnitude, response time and recovery time as the performance indicators of a gas sensor device.

\section{Computational techniques}

The processing unit of artificial neural network (ANN) bears a resemblance to that of a human brain. Inputs generated from other processing units are accepted by the multiple layered processing units that constitute the network architecture. The concluding output of the scheme is the productivity that is generated on the completion of processing from different units. The processing and modelling of materials are provided a profoundly sound and innovative approach through the ANN's. ANN can recognize the outline of inputs and outputs commencing the earlier encounters and even the present forms without any previous assumption of their characteristics and interconnections, and this is the most important characteristic of ANN. When compared with the traditional approaches, the network has the capacity to calculate and determine further difficult relations in the data of material properties. A quantity of inputs (experimental variables), an only output, and an inbetween hidden layer are contained by the network. At individual hidden unit $\left(h_{i}\right)$, the weighted amalgamation of the standardized inputs $\left(x_{j}^{N}\right)$ is functioned on a hyperbolic tangent transfer function which is presented in Eq. (1), creates definite that each input subsidizes to each and every hidden unit.

$$
h_{i}=\tanh \left(\sum_{j} w_{i j}^{(1)} x_{j}^{N}+\theta_{j}^{(1)}\right)
$$

After that, output neuron at that time computes a linear weighted sum of the outputs of the hidden units, as indicated in Eq. (2):

$$
y=\sum_{i} w_{i}^{(2)} h_{i}+\theta^{2}
$$

In the directly above for both equations, $y$ is the output, $x_{j}^{N}$ defined as normalized inputs, $h_{i}$ defined as the outputs through hidden units, $w_{i j}, w_{i}$ defined as weights, and $\theta_{i}$ and $\theta$ defined as bias. Therefore, which is likely to achieve dissimilar outputs via changing the weights, $w_{i j}$ ((Equations (1) and (2)). The optimal values of these weights are achieving through "training" the network on a set of normalized inputoutput data. For that, the input-output data can be first standardized in the sort of -1 to +1 from the Eq. (3):

$$
x_{j}^{N}=\frac{\left(x_{j}-x_{\min }\right) \times 2}{x_{\max }-x_{\min }}-1
$$


Designing optimized ternary catalytic alloy electrode for efficiency improvement of semiconductor gas sensors using a machine learning approach

Here $x_{j}^{N}$ is denoted by normalized value, $x_{j}$ signifies the input or output variable, $x_{\min }$ and $x_{\max }$ are the minimum and the maximum values of the variable, respectively. The network is trained by adjusting the weights $\left(W_{i j}\right)$ to minimize an error function, which is basically a regularized sum of square errors. This ultimately leads to an optimal description of the input-output relationship.

Table 2. Parameters used in the multi-objective genetic algorithm

\begin{tabular}{cc}
\hline Crossover probability & 0.95 \\
\hline Random seed value & 0.19 \\
\hline Mutation probability & 0.05 \\
\hline Parental Selection Strategy & Tournament selection \\
\hline
\end{tabular}

For resolving the back propagation (BP) algorithm, is intended of determination of weights and biases for a multilayer ANN using feed forward connections through the input layer to the hidden layers and then to the output layer. For minimizing the mean square error among the projected output and the preferred output, the algorithm is an iterative gradient lineage algorithm, which is intended. Scaled conjugate gradient back propagation algorithm was used for the contemporary work. Via determination, the effect of the input parameters on the final things is impossible, as artificial neural network (ANN) is a very high compound and non-linear model.

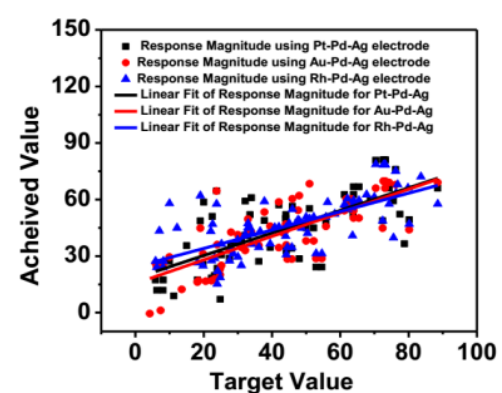

(a)

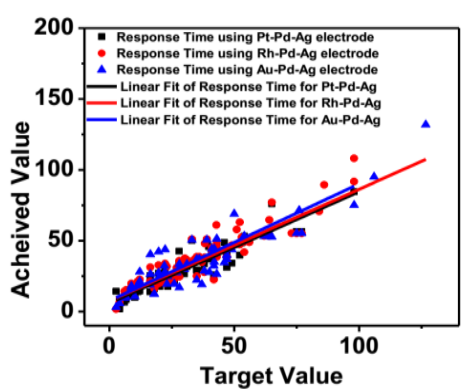

(b)

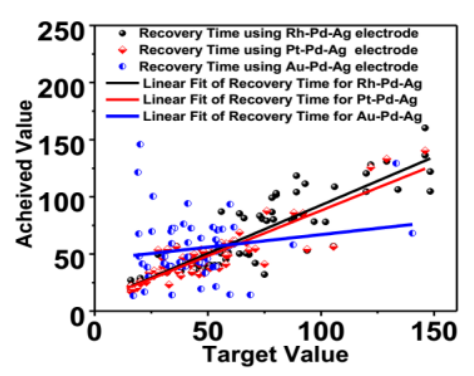

(c)

Figure 1. Representative scatter plot showing the prediction by the trained ANN models for (a) response magnitude for the three alloy combinations under consideration (b) response time for the three alloy combinations under consideration and (c) recovery time for the three alloy combinations under consideration. 

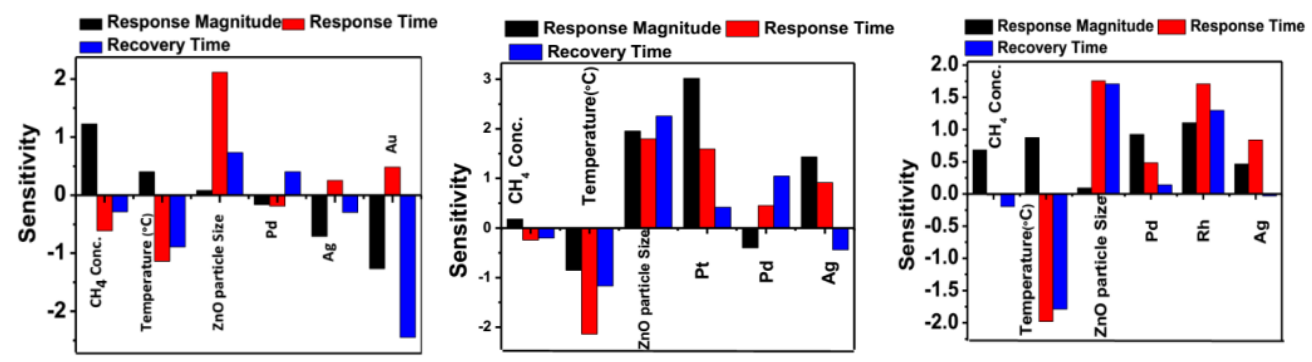

Figure 2. Sensitivity studies of the independent variables for all three output parameters, For (a) Au-Pd-Ag, (b) Pt-Pd-Ag, (c) Rh-Pd-Ag alloys.

This makes it imperative to conduct a sensitivity study to reveal the complex hidden connection in the ANN model. Sensitivity analysis would reveal the gross virtual prominence of the parameters on the belongings. Researchers have used several approaches of sensitivity study. For this work, the connection weight method was selected (DOlden, 2004). To compute the sensitivity, weights of the input-hidden and hidden-output associates in the competent ANN models have been used. The philosophy of advancement of classes promoted by Charles Darwin has inspired the improvement of the unconventional optimization technique recognized as Genetic algorithm (GA). Selection, crossover and mutation are the main biological principles which are followed via the Simple Genetic Algorithm (SGA) (Pławiak \& Rzecki, 2015; Singh et al., 2020). In Case of selection process, the candidates aimed at next generation which were recognized. Through the crossover process via exchanging and transmission of genetic statistics among two parents for the birth of offspring is transported. Finally, a small, probabilistic variation in the genetic makeup is made by the mutation process. In another circumstance, one of the objective conflicting in character, then method is termed as multi-objective optimization (MO0) (Malbašić \& Đurić, 2020). Unlike single objective optimization where a single prime solution evolves, in circumstance of MOO, a non-dominated set of solutions, termed as the 'Pareto front', evolve (Dey et al., 2015; Milosevic et al., 2021). This enables a decision producer to select the utmost suitable solution out of the numerous alike finest solutions trading off amongst the differing objectives. In the present work, the developed ANN models which are describing the three features of the sensor system are recycled as the objective functions and Non-Dominated Sorting Genetic Algorithm (NSGA-II) code (Deb et al., 2002; Albu et al., 2019; Gharib, 2020; Messinis \& Vosniakos, 2020), while using for the multi-objective optimization. The constraints used for optimization are specified in Table 2 .

\section{Results and Discussion}

Figure1 displays the performances of some of the developed artificial neural network (ANN) models for predicting response magnitude, response time and recovery time for all the three alloy combinations under consideration. The target versus achieved output plots for the ANN models show that the performance of most of the ANN models are satisfactory. But in some cases, particularly for the response magnitude models, the prediction by the ANN models are not as expected. The sensitivity analyses of the variables based on the trained ANN models are shown in Fig. 2. It is seen that in the Pd-Ag-Au alloy, $\mathrm{Au}$ has the most significant role in reducing the recovery time. The role of the other alloying elements is not that 
Designing optimized ternary catalytic alloy electrode for efficiency improvement of semiconductor gas sensors using a machine learning approach

significant in improving the output parameters of the sensor device. While, the other two ternary alloy systems, Pt in Pt-Pd-Ag alloy and Pd and Rh in Pd-Rh-Au alloy seem to have positive effect in increasing the response magnitude. The multi-objective optimization using genetic algorithm generates non-dominated solutions in the form of 'Pareto front'. The Pareto solutions are generated with different combinations of objectives, viz. (i) response magnitude and response time, (ii) response magnitude and recovery time, and (iii) response magnitude, response time and recovery time for designing all three alloy systems for three different $\mathrm{ZnO}$ particle sizes separately. Different combinations of objective functions are used for the multi-objective optimization processes to study the role of the variables in the optimum solutions for different conflicting situations. The results from the optimizations taking three objectives together provide the solutions which can be processed for further investigations, even experimental trials, as those consider all the factors required for the improvement of the catalytic alloy performance.

Some representative results are selected for all three alloy systems and shown as Fig. 3 and Fig. 4 and Tables 3-5.

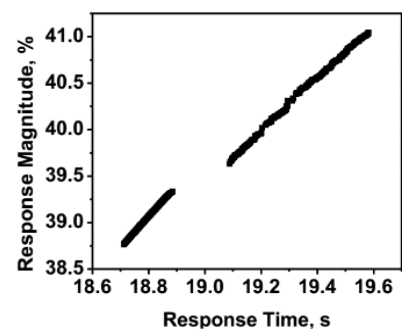

(a)

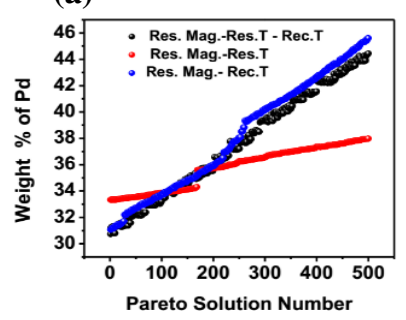

(d)

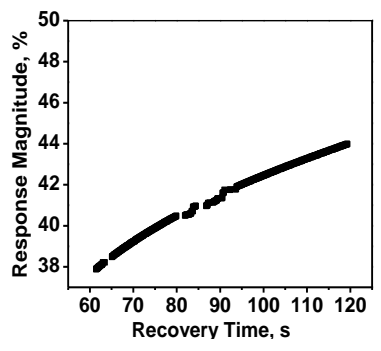

(b)

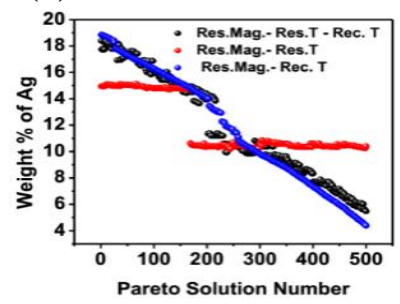

(e)

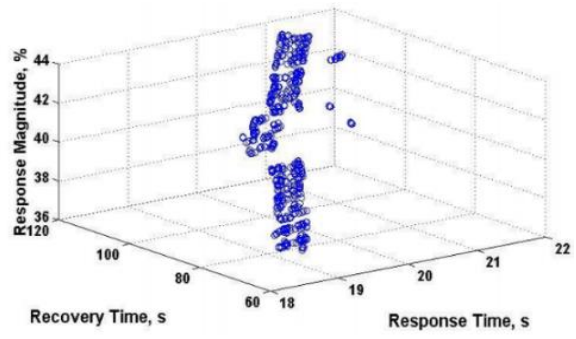

(c)

Figure 3. The multi-objective optimization results for the Au-Pd-Ag alloy (a) Pareto front for response magnitude and response time, b) Pareto front for response magnitude and recovery time, (c) Pareto front for response magnitude, response time and recovery time, (d) Variation of Pd in the Pareto solution with increasing response magnitude, and (e) Variation of $\mathrm{Ag}$ in the Pareto solution with increasing response magnitude for different search processes.

Fig. 3(a-c) shows the Pareto fronts of Au-Pd-Ag alloy system generated from multi-objective optimization of different combinations of objectives for $\mathrm{ZnO}$ particle size of $45 \mathrm{~nm}$. The conflicting nature of the two objectives are clearly visible when response magnitude is maximized with simultaneous minimization of recovery time 
(Fig. 3b). The conflict between the objectives of maximizing response magnitude with minimization of response time is not that significant, as evident from the spread of the Pareto front (Fig. 3a). The same phenomenon can be observed in the Pareto surface developed using all three objectives together (Fig. 3c). The non-dominated solutions for all three cases of optimization are arranged along with increasing response magnitude and numbered.

The maximum and minimum values of the amounts of elements used in the ternary alloys with optimized performance, as shown in the Pareto solutions for all three optimization conditions, are described in Table 3.

It is seen that the $\mathrm{Au}$ content does not vary significantly for any optimization condition and remains close to $50 \mathrm{wt} \%$. The other two elements, i.e. Pd and $\mathrm{Ag}$, have varied to a certain extent during tradeoff between the objectives. The variation in $\mathrm{Pd}$ and Ag for the bi-objective optimization of response magnitude and response time are lesser than the other two cases. This is in conformance of the finding of the Pareto front (Fig. 3a). The variations of the Pd and $\mathrm{Ag}$ in the solutions for three different optimization conditions are plotted in Figs. 3(d-e), where increasing number of Pareto solution depicts increase in response magnitude.

Table 3. The ranges of the weight percentages of the elements in the optimum ternary (Au-Pd-Ag) alloys in the Pareto solutions of three different optimization process.

\begin{tabular}{ccccccc}
\hline Objectives & \multicolumn{2}{c}{ Au (wt\%) } & \multicolumn{2}{c}{ Pd (wt\%) } & \multicolumn{2}{c}{ Ag (wt\%) } \\
\hline & Minimum & Maximum & Minimum & Maximum & Minimum & Maximum \\
\hline RM -Res.T & 50.93941 & 53.87713 & 33.33568 & 37.9842 & 10.20336 & 15.14193 \\
\hline R.M -Rec.T & 50 & 50.53885 & 31.07032 & 45.603958 & 4.375566 & 18.855211 \\
\hline $\begin{array}{c}\text { RM -Res.T-Rec. } \\
\text { T }\end{array}$ & 50.00015 & 52.65257 & 30.72845 & 44.444019 & 5.47625 & 18.394596 \\
\hline
\end{tabular}

Table 4. The ranges of the weight percentages of the elements in the optimum ternary (Pt-Pd-Ag) alloys in the Pareto solutions of three different optimization process.

\begin{tabular}{ccccccc}
\hline Objectives & \multicolumn{2}{c}{ Pt $(\mathrm{wt} \%)$} & \multicolumn{2}{c}{ Pd $(\mathrm{wt} \%)$} & \multicolumn{2}{c}{ Ag (wt $\%)$} \\
\hline & Minimum & Maximum & Minimum & Maximum & Minimum & Maximum \\
\hline RM -Res.T & 50 & 50.00064 & 30 & 30.000008 & 20.09934 & 20.099768 \\
\hline R.M -Rec.T & 50.00336 & 50.00866 & 30 & 30.000065 & 20.09123 & 20.091375 \\
\hline RM -Res.T-Rec. T & 50.0002 & 50.10892 & 30 & 30.000731 & 19.98956 & 20.09416 \\
\hline
\end{tabular}

It is clear from the figure that increase in Pd with simultaneous decease in $\mathrm{Ag}$ increase the response magnitude, with the expense of increase in recovery and response times. The trends of disparity of $\mathrm{Ag}$ and $\mathrm{Pd}$ in the Pareto solution are same in the bi-objective optimization response magnitude and recovery time and the triobjective optimization for all objectives. This gives clear indication that to achieve a certain performance level, i.e. to achieve a specific tradeoff between the three properties, the user can get the required alloy composition easily from those to Pareto solutions. The other Pareto solution, developed from optimization of response magnitude and response time, may provide a different solution to some extent. The minimum and the maximum values of the alloying elements in the Pareto solutions of 
Designing optimized ternary catalytic alloy electrode for efficiency improvement of semiconductor gas sensors using a machine learning approach

Pt-Pd-Ag alloy system for $\mathrm{ZnO}$ particle size of $60 \mathrm{~nm}$ are given in Table 4 . The values clearly indicate that the optimum weight percentages for the three elements are almost same throughout the Pareto front, and even for all three types of multiobjective optimizations

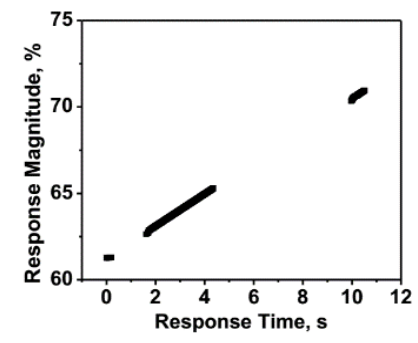

(a)

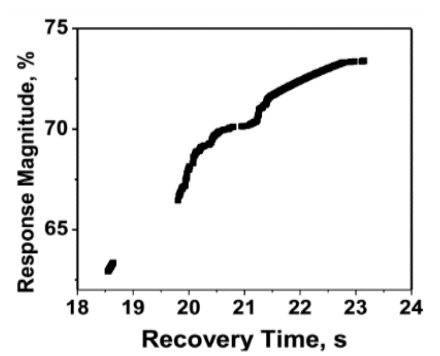

(b)

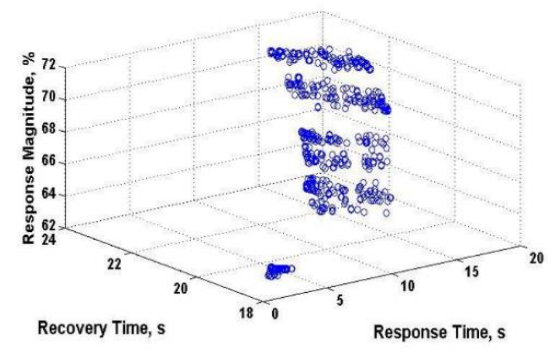

(c)

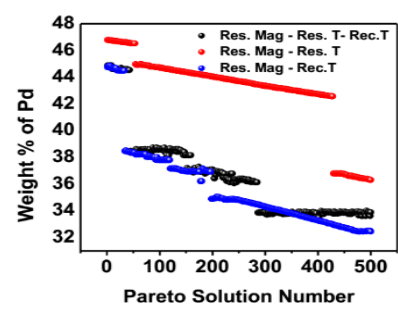

(d)

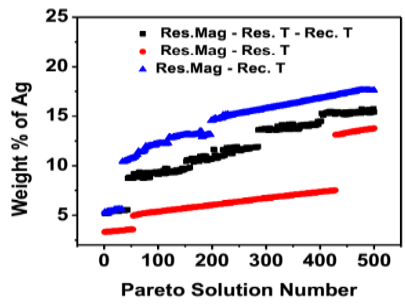

(e)

Figure 4. The multi-objective optimization results for the Rh-Pd-Ag alloy (a) Pareto front for response magnitude and response time, b) Pareto front for response magnitude and recovery time, (c) Pareto front for response magnitude, response time and recovery time, (d) Variation of Pd in the Pareto solution with increasing response magnitude, and (e) Variation of Ag in the Pareto solution with increasing response magnitude for different search processes.

For all cases a Pt0.5Pd0.3Ag0.2 alloy provides the best performance. This leads to the fact that the three objectives do not have any significant conflict between them, at least within the used search space. Some of the important results coming out from the multi-objective optimizations for the Rh-Pd-Ag alloy system for $\mathrm{ZnO}$ particle size 30 $\mathrm{nm}$ are shown in Fig.4. The Pareto fronts of the alloy system generated from three different optimization processes, as before, are given in Fig. 4(a-c).

Table 5. The ranges of the weight percentages of the elements in the optimum ternary (Rh-Pd-Ag) alloys in the Pareto solutions of three different optimization process

\begin{tabular}{ccccccc}
\hline Objectives & \multicolumn{2}{c}{ Rh (wt\%) } & \multicolumn{2}{c}{ Pd (wt\%) } & \multicolumn{2}{c}{ Ag (wt\%) } \\
\hline & Minimum & Maximum & Minimum & Maximum & Minimum & Maximum \\
\hline RM -Res.T & 50 & 50 & 36.32003 & 46.794388 & 3.304113 & 13.77707 \\
\hline R.M -Rec.T & 50 & 51.16267 & 32.45868 & 44.862404 & 5.232672 & 17.638802 \\
\hline $\begin{array}{c}\text { RM - Res.T-Rec. } \\
\text { T }\end{array}$ & 50 & 52.85969 & 33.32817 & 44.906982 & 5.137318 & 115553006 \\
\hline
\end{tabular}


It is seen in this case that the variations of the properties are quite high in the nondominated solution, which means the objectives are quite conflicting in this alloy. Table 5 gives the ranges of the alloying elements required to achieve optimum solutions for improved performance, as shown in the Pareto solutions of the three optimizations. Here it is seen again that the major element of this alloy (Rh) has not varied for all the solutions and remained at around $50 \mathrm{wt} \%$, same as previous two alloy systems. When the variations of other two elements in the solutions are plotted, it is seen that $\mathrm{Pd}$ content decreases and $\mathrm{Ag}$ increases with increasing in response magnitude (Fig. 4(d-e)). This is contrary to the trend shown in Au-Pd-Ag alloy. The amount of Pd for different trade off options the Pareto front shows almost similar values in case of optimization of response magnitude and recovery time as well as in case where all three objectives are considered together. But the solutions from the optimization of response magnitude and response time show higher values of $\mathrm{Pd}$. In case of addition of Ag as the third element, the trend is similar for the two cases as above, whereas the third optimization expectedly shows lower values of Ag.

The results of the data-driven modelling using ANN and multi-objective optimization using GA for designing three alloys, viz. Au-Pd-Ag, Pt-Pd-Ag and Rh-Pt$\mathrm{Ag}$, have shown some clear trends in most of the cases. In the earlier work (Ghosal et al., 2019), the GA based searching for ternary alloys with improved performance was completely random in nature, which was necessary to gather primary idea about the probable set of ternary alloys. But in this work, a systematic search has been carried out, where the prior knowledge regarding the role of the various elements has been incorporated meticulously in the searching process. In the process the three alloy systems have one base element each ( $\mathrm{Au}, \mathrm{Pt}$ and $\mathrm{Rh}$ ), one major alloying element (Pd) and one addition of comparatively lower amount $(\mathrm{Ag})$. This makes the variations in compositions more perceptible, compared to the randomness in variation of composition previously, and thus the decision-making process for experimental trial becomes more logical. During the experimental validation, one should remember that all the results reported here based on data-driven models generated from data collected from secondary source, and hence it is better to consider the general trends of the findings, not a particular solution.

\section{Conclusion}

Catalytic noble metals in the form of electrode element were found to improve the semiconductor gas sensor device performance. Earlier experimental findings revealed that alloy of such noble metals improved the sensing parameters, viz. response magnitude, response time, recovery time, operating temperature and selectivity. However, optimizing all these parameters simultaneously is a crucial challenge, as the requirement for such individual optimization is often mutually conflicting. In the present paper, a systematic approach based on ANN and GA was reported to design an optimized ternary alloy electrode from the constructed database of the earlier reported binary ones. It was found with such GA based multiobjective optimization, the gas sensor device performance can be judiciously optimized employing the properly designed ternary alloy, as the electrode material. It was found that, Pt-Pd-Ag (wt \% ratio of Pt: 50\%, Pd: $30 \%$, Ag: 20\%) offered most promising results for output parameters among the lot. The experimental verification for the present theoretical simulation has been considered as future work. 
Designing optimized ternary catalytic alloy electrode for efficiency improvement of semiconductor gas sensors using a machine learning approach

Acknowledgement: This publication is a product of the Research and Development work commenced in the project under the Visvesvaraya Ph.D. Scheme of Ministry of Electronics and Information Technology, Government of India, being instigated by Digital India Corporation (formerly Media Lab Asia).

Author Contributions: Each author has participated and contributed sufficiently to take public responsibility for appropriate portions of the content.

Funding: This research received no external funding.

Conflicts of Interest: The authors declare no conflicts of interest.

\section{References}

Acharyya, D. \& Bhattacharyya, P. (2016). Alcohol sensing performance of ZnO hexagonal nanotubes at low temperatures: A qualitative understanding. Sens. Actuators B, Chem. 228, 373-386, doi: 10.1016/j.snb.2016.01.035.

Acharyya, D., Huang, K. Y. , Chattopadhyay, P. P., Ho, H. M. S., Fecht J. \& Bhattacharyya, P. (2016). Hybrid 3D structures of ZnO nanoflowers and PdO nanoparticles as a highly selective methanol sensor. Analyst, 141, 2977-2989, doi: 10.1039/ c6an00326e.

Albu, A., Precup, R.E., Teban, T.A. (2019). Results and challenges of artificial neural networks used for decision-making and control in medical applications. Facta universitatis series: Mechanical Engineering, 17(3), 285 - 308.

Anderson, J. A. (1995). An Introduction to Neural Networks. MIT Press, Cambridge MA.

Basu, P. K., Bhattacharyya, P., Saha, N., Saha, H. \& Basu S. (2008). The superior performance of the electrochemically grown $\mathrm{ZnO}$ thin films as methane sensor. Sensors and Actuators B, 133 (2), 357-363.

Bhattacharyya, P., Basu, P. K., Lang, C., Saha, H. \& Basu S. (2008). Noble metal catalytic contacts to sol-gel nanocrystalline zinc oxide thin films for sensing methane. Sensors and Actuators B, 129 (2), 551-557.

Bhattacharyya, P., Basu, P. K., Lang, C., Saha, H. \& Basu, S. (2008). Noble metal catalytic contacts to sol gel nanocrystalline zinc oxide thin films for sensing methane. Sensors and Actuators B, 129 (2), 551-557.

Bhattacharyya, P., Basu, P. K., Mukherjee, N., Mondal, A., Saha, H. \& Basu S. (2007). Deposition of nanocrystalline $\mathrm{ZnO}$ thin films on $\mathrm{p}$-Si by novel galvanic method and application of the heterojunction as methane sensor. Journal of Materials Science: Materials in Electronics, 18 (8), 823-829.

Bhattacharyya, P., Basu, P. K., Saha H. \& Basu, S. (2007). Fast response methane sensor using nanocrystalline zinc oxide thin films derived by sol-gel method. Sensors and Actuators B, 124 (1), 62-67. 
Bhattacharyya, P., Bhowmik, B. \& Fecht, H. J. (2015). Operating Temperature, Repeatability, and Selectivity of $\mathrm{TiO}_{2}$ Nanotube-Based Acetone Sensor: Influence of Pd and $\mathrm{Ni}$ Nanoparticle Modifications. IEEE Transactions on Device and Materials Reliability, 15(3).

Datta, S. \& Chattopadhyay, P. P. Soft Computing Techniques in Advancement of Structural Metals.(2013). International Materials Reviews, 58 (8), 475-504.

Datta, S. (2016). Materials Design Using Computational Intelligence Techniques, CRC Press.

Deb K . (2001).Multi-Objective Optimization Using Evolutionary Algorithms. John Wiley \& Sons Ltd., Chichester.

Deb, K., Pratap, A., Agarwal, S. \& Meyarivan T. (2002). A fast and elitist multiobjective genetic algorithm: NSGA-II. IEEE Trans., Evolutionary Computation, 6, 182-19

Dey, S., Ganguly, S. \& Datta, S. (2015). In silico design of high strength aluminium alloy using multi-objective GA. Springer International Publishing Switzerland, doi https://doi.org/10.1007/978-3-31920294-5_28.

Dey. S., Sultana, N., Kaiser, Md., S., Dey, P. \& Datta, S. (2016). Computational intelligence based design of age-hardenable aluminium alloys for different temperature regimes. Materials and Design, 92, 522-534.

Dolden, J., KJoy, M. \& GDeath, R. (2004). An accurate comparison of methods for quantifying variable importance in artificial neural networks using simulated data. Ecological Modelling. 178 (3-4), 389-397.

Gharib, M. R. (2020). Comparison of robust optimal QFT controller with TFC and MFC controller in a multi-input multi-output system. Reports in Mechanical Engineering, 1(1), 151-161.

Ghosal, S., Dey, S., Bhattacharyya, P., Chattopadhyay, P. P., Datta, S. (2019). Datadriven design of ternary alloy catalysts for enhanced oxide-based gas sensors' performance. Computational Materials Science, 161, 255-260.

Goldberg D. E. (2002). Genetic Algorithms in Search, Optimization and Machine Learning. Pearson-Education, New Delhi.

Kumar, R., Das, R. R., Mishra, V. N. \& Dwivedi R. (2011). Wavelet Coefficient Trained Neural Network Classifier for Improvement in Qualitative Classification Performance of Oxygen-Plasma Treated Thick Film Tin Oxide Sensor Array Exposed to Different Odors/Gases. IEEE Sensors Journal, 11 (4).

Kumar, S. (2004). Neural Network-A Classroom Approach. Tata McGraw-Hill Publishing Company Limited, New Delhi.

Lee, S. M. , Dyer, D. C. \& Gardner J W. (2003). Design and optimisation of a hightemperature silicon micro-hotplate for nanoporous palladium pellistors. Microelectronics Journal, 34 (2), 115-126.

Longo, G. A., Zilio, C., Ortombina, L. \& Zigliotto, M. (2017). Application of Artificial Neural Network (ANN) for modeling oxide-based nanofluids dynamic viscosity. International Communications in Heat and Mass Transfer, 83, 8-14. 
Designing optimized ternary catalytic alloy electrode for efficiency improvement of semiconductor gas sensors using a machine learning approach

Maity, I., Acharyya, D., Huang, K., Chung, P., Ho, M. \& Bhattacharyya, P. (2018). A Comparative Study on Performance Improvement of ZnO Nanotubes Based Alcohol Sensor Devices by Pd and rGO Hybridization. IEEE Transactions on Electron Devices, 65( 8), 3528-3534.

Malbašić, S.B., \& Đurić, S.V. (2019). Risk assessment framework: Application of Bayesian Belief Networks in an ammunition delaboration project. Military Technical Courier, 67(3), 614-641.

Messinis, S., \& Vosniakos, G. C. (2020). An agent-based flexible manufacturing system controller with Petri-net enabled algebraic deadlock avoidance. Reports in Mechanical Engineering, 1(1), 77-92.

Milosevic, T., Pamucar, D., \& Chatterjee, P. (2021). Model for selecting a route for the transport of hazardous materials using a fuzzy logic system. Military Technical Courier, 69(2), 355-390.

Mishra, G. P., Sengupta, A., Maji, S., Sarkar, S. K. \& Bhattacharyya, P. (2010). The Effect of Catalytic Metal Contact on Methane Sensing Performance of Nanoporous ZnO -Si Heterojunction. International Journal on Smart Sensing and Intelligent Systems, 3 (2).

Pławiak, P. \& Rzecki K. (2015). Approximation of Phenol Concentration Using Computational Intelligence Methods Based on Signals from the Metal-Oxide Sensor Array. IEEE Sensors Journal, 15(3).

Quaranta, F., Rella, R., Siciliano, P., Capone, S., Epifani, M. \& Vasanelli, L. (1999). A novel gas sensor based on $\mathrm{SnO}_{2} / \mathrm{Os}$ thin film for the detection of methane at low temperature. Sensors and Actuators B., 58, 350-355.

Ray, M., Ganguly, S., Das, M., Datta, S., Bandyopadhyay, N. R. \& Hossain, S. M. (2009). ANN based model for in situ prediction of porosity of nanostructured porous silicon. Materials and Manufacturing Processes, 24 (1), 83-87.

Roy, S., Sarkar, C. K. \& Bhattacharyya, P. (2012). A highly sensitive methane sensor with nickel alloy microheater on micromachined Si substrate. Solid-State Electronics, $2,76,84-90$.

Singh, A., Ghadai, R.K., Kalita, K., Chatterjee, P., Pamucar, D. (2020). EDM process parameter optimization for efficient machining of Inconel-718. Facta Universitatis, series: Mechanical Engineering. 18(3), 473 - 490.

Sinha, A., Sikdar, S. (Dey), Chattopadhyay,P. P. \& Datta, S. (2013). Optimization of mechanical property and shape recovery behavior of Ti-(_49 at \%) Ni alloy using artificial neural network and genetic algorithm. Materials and Design, 46, 227-234.

Wollenstein, J., Burgmair, M., Plescher, G.,Sulima, T., Hildenbrand, J. \& Bottner, H. (2003). Cobalt oxide based gas sensors on silicon substrate for operation at low temperatures. Sensors and Actuators B, 93 (1-3), 442-448.

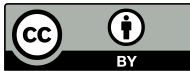

(C) 2018 by the authors. Submitted for possible open access publication under the terms and conditions of the Creative Commons Attribution (CC BY) license (http://creativecommons.org/licenses/by/4.0/). 